\title{
铜促进硫代色酮类化合物的合成研究
}

\author{
李明宁加涁于乐文丽荣*
}

(青岛科技大学化学与分子工程学院 生态化工国家重点实验室培育基地 青岛 266042)

\begin{abstract}
摘要 基于底物设计的概念, 建立了一种以 $\beta$-羰基二硫代羧酸酯和取代邻溴代苯乙酮为合成子, 在碘化亚铜/邻菲罗啉 促进下高化学选择性地合成硫代色酮类衍生物的新方法. 该方法高效、简便且收率高.

关键词 硫代色酮; $\beta$-羰基二硫代羧酸酯; 取代邻溴苯乙酮; 铜; Ullmann 偶联反应; 合成
\end{abstract}

\section{Copper-Promoted Synthesis of Thiochromones Derivatives}

\author{
Li, Ming Ning, Jiabin Yu, Le Wen, Lirong* \\ (State Key Laboratory Base of Eco-Chemical Engineering, College of Chemistry and Molecular Engineering, \\ Qingdao University of Science and Technology, Qingdao 266042)
}

\begin{abstract}
A new methodology to high chemoselectively synthesize thiochromone derivatives from $\beta$-oxodithioesters and substituted $o$-bromoacetophenones promoted by $\mathrm{CuI} / 1,10$-phen system based on the substrate-design concept has been developed. This approach has advantages of simple and efficient operation, and high yields.

Keywords thiochromones; $\beta$-oxodithioesters; substituted $o$-bromoacetophenones; copper; Ullmann coupling reaction; synthesis
\end{abstract}

硫代色酮是苯并吡喃酤的硫代同系物，是一类具有 潜力的候选药物. 研究表明, 该类化合物易于通过真菌 的细胞膜, 改变真菌细胞的超微结构, 进而破坏其细胞 膜及细胞壁的结构与功能, 引起细胞内容物流出而导致 真菌死亡. 因此, 许多硫代色酮类化合物都表现出抗 菌 $^{[1]}$ 、抗病毒 ${ }^{[2]}$ 等活性. 一些衍生物被用作抗疮疾药物 ${ }^{[3]}$, 具有可逆地抑制人体巨细胞病毒的功能 ${ }^{[4]}$. 另外, 此类 化合物还可作为关键中间体用来合成其它具有生物活 性的化合物 ${ }^{[5]}$ 以及光不稳定化合物的保护剂 ${ }^{[6]}$. 尽管硫 代色酮的合成方法已有很多文献报道 ${ }^{[7]}$, 但往往存在一 些缺限. 例如, 使用酰氯、酸或强碱, 反应条件较为苛 刻, 步骤繁琐且收率不高等.

在过去的几十年中, 关于铜催化卤代芳基化合物的 偶联反应备受关注. 由于铜催化剂往往比钯、铑、镍等 金属催化剂价廉低毒, 对环境相对更友好, 现已成为有 效构建 $\mathrm{C}-\mathrm{C}$ 和 $\mathrm{C}-\mathrm{X}(\mathrm{N}, \mathrm{O}, \mathrm{S}, \mathrm{Se}$ 等)键的一种重要手段 ${ }^{[8]}$.

$\beta$-羰基二硫代羧酸酯是一种非常有用的多官能团
合成子, 具有 1,3-二羰基化合物类似的性质. 过去几十 年 $\beta$-羰基二硫代羧酸酯已经被广泛应用在合成各种含 硫化合物中 ${ }^{[9]} . \beta$-羰基二硫代羒酸酯存在两个亲电和三 个亲核反应活性中心, 可以与各种双亲电试剂反应，根 据双亲电试剂的性质结构的不同、反应条件的不同可形 成不同的含硫杂环或含硫取代基杂环化合物. 本课题组 在近年来以 $\beta$-羰基二硫代羧酸酯为合成子的各种反应 研究基础上 ${ }^{[10]}$, 建立了一种碘化亚铜促进 $\beta$-羰基二硫代 羧酸酯和取代邻溴苯乙酮发生 Ullmann 偶联反应，高效 快速地合成 2-(2-氧代-2-芳乙基)- $4 H$-苯并噻喃-4-酮衍生 物的新方法.

\section{1 结果与讨论}

$\beta$-羰基二硫代羧酸酯与不同的双官能团试剂反应 时, 反应产物中硫原子可能存在于环内或者环外, 形成 不同的杂环结构. 本研究中, $\beta$-羰基二硫代羧酸酯与邻 澳苯乙酮在铜促进下可能存在两种偶联模式(Scheme $1)$.

\footnotetext{
* E-mail: wenlirong@qust.edu.cn

Received May 17, 2016; revised June 12, 2016; published online July 7, 2016
}

Project supported by the National Natural Science Foundation of China (Nos. 21572110, 21372137) and the Natural Science Foundation of Shandong Province (No. ZR2014BM006).

国家自然科学基金(Nos. 21572110, 21372137)和山东省自然科学基金(No. ZR2014BM006)资助项目. 


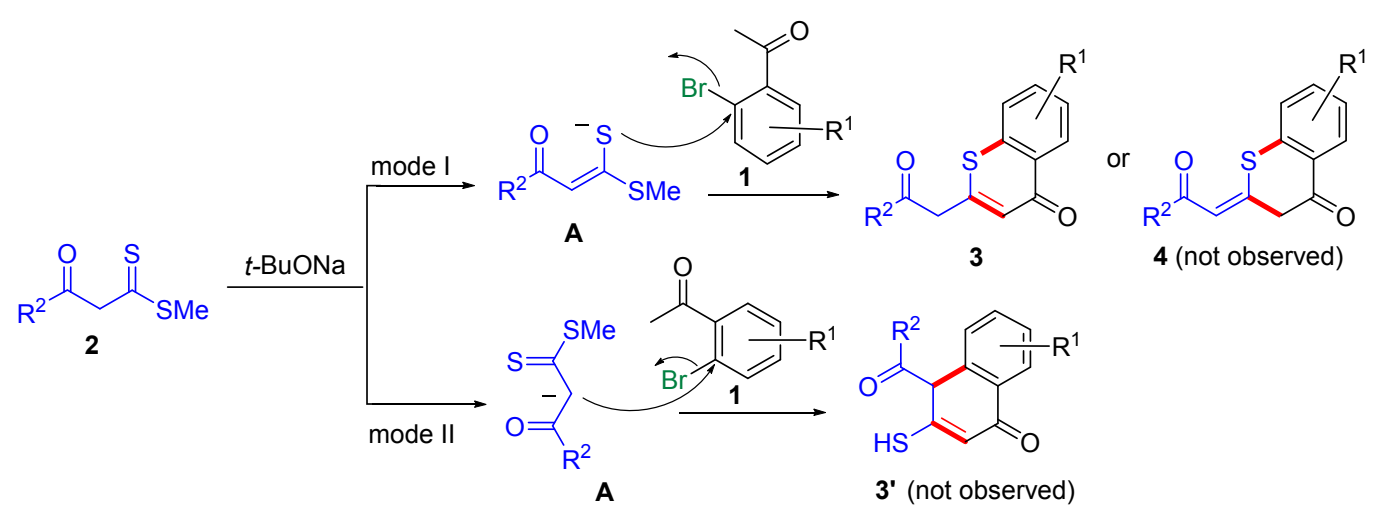

图式 1 底物 2 与 1 反应的化学选择性

Scheme 1 Chemoselectivity of the reaction of substrates $\mathbf{2}$ with 1

在模式 I 中, 底物 2 的硫羰基中硫原子与取代邻溴 苯乙酮 1 经 Ullmann 偶联反应和分子内亲核取代反应得 到苯并噻喃-4-酮类化合物 3 或 4; 在模式 II 中, 偶联发 生在 $\beta$-羰基二硫代羧酸酯的亚甲基上, 经 Hurtley 偶联 反应 ${ }^{[10 a]}$ 得到 3-颈基荎-1-(4H)-酮类化合物 $\mathbf{3}^{\prime}$. 通过重水 交换核磁共振分析排除了产物 3'; 但是由于化合物 3 和 4 互为同分异构体且结构相似，通过简单的核磁氢谱、 碳谱和质谱检测手段难以鉴定其结构, 且多次单晶培养 均未成功. 最终通过化合物 3d 的二维核磁共振 HMBC 谱图分析确定了产物结构为 $\mathbf{3}$ (图 1).
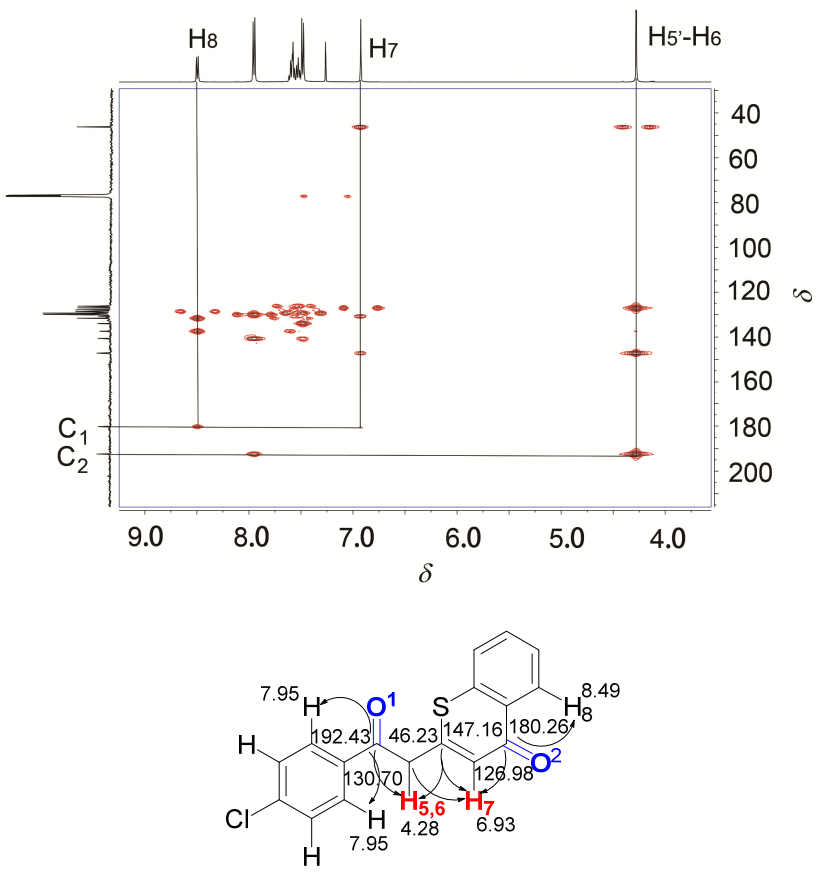

图 1 化合物 3d 的 $\mathrm{HMBC}$ 谱图及 ${ }^{1} \mathrm{H}-{ }^{13} \mathrm{C}$ 远程偶合关系(部分) Figure 1 HMBC spectrum and ${ }^{1} \mathrm{H}^{13}{ }^{13} \mathrm{C}$ long-range couple correlation of compound $\mathbf{3 d}$ (part)

根据化合物 3d 的 ${ }^{1} \mathrm{H}$ NMR 和 ${ }^{13} \mathrm{C}$ NMR 谱图可知, 在图 1 中, $\delta_{\mathrm{H}} 4.28$ 处为饱和氢 $\mathrm{H}^{5} 、 \mathrm{H}^{6}$ 的吸收峰, $\delta 6.93$
处为 $\mathrm{C}=\mathrm{C}-\mathrm{H}$ 氢 $\mathrm{H}^{7}$ 的吸收峰, $\delta 8.49$ 处氢为 $\mathrm{H}^{8}$ 的吸收 峰; $\delta 180.26$ 处是取代邻溴苯乙酮骨架中的羰基碳, $\delta$ 192.43 处是 $\beta$-羰基二硫代羧酸酯骨架中羰基碳. 由 $\mathrm{HMBC}$ 谱图可知 $\mathrm{H}^{8}$ 与 $\delta 180.26$ 羰基碳偶合, 化合物饱 和 $\mathrm{H}^{5} 、 \mathrm{H}^{6}$ 与 $\delta 192.43$ 处羰基碳远程相关, 烯烃氢 $\mathrm{H}^{7}$ 与 $\delta 180.26$ 处羰基碳远程相关, 从而确定目标化合物结构 为 3 而非 4. 更详细的解析见表 1. 此谱图分析结果与文 献报道的该化合物结构一致 ${ }^{[11]}$.

表 1 化合物 3d 的 HMBC 谱图解析(部分)

Table 1 The analysis of the HMBC spectrum of compound $\mathbf{3 d}$ (part)

\begin{tabular}{cccccl}
\hline 序号 & $\delta_{\mathrm{H}}$ & 氢数 & 峰型 & $J / \mathrm{Hz}$ & \multicolumn{1}{c}{ 远程相关的 $\delta_{\mathrm{C}}$} \\
\hline 1 & 4.28 & 2 & $\mathrm{~s}$ & & $126.98,147.16,192.43$ \\
2 & 6.93 & 1 & $\mathrm{~s}$ & & $46.23,147.16,130.70$ \\
& & & & & 180.26 \\
3 & 7.48 & 2 & $\mathrm{~d}$ & 8.50 & $129.30,129.91,133.92$ \\
4 & 7.95 & 2 & $\mathrm{~d}$ & 8.55 & $129.30,140.66,192.43$ \\
5 & 8.49 & 1 & $\mathrm{~d}$ & 8.05 & $131.60,137.40,180.26$ \\
\hline
\end{tabular}

\section{1 最佳反应条件的选择}

以邻溴苯乙酮(1a)和苯甲酰基二硫代乙酸甲酯(2a) 的反应为模型反应，分别对铜催化剂、配体、碱、溶剂、 温度等反应条件进行了笁选，结果见表 2 .

首先，该反应在甲苯溶剂中 $80{ }^{\circ} \mathrm{C}$ 条件下，不添加 催化剂和碱、或有催化剂无碱、或无催化剂只加碱时，均 没有产物生成(表 2, Entries 1 3). 令人高兴的是, 当同 时加入 1 倍量的 $\mathrm{CuI}$ 作催化剂(无配体)和 4 倍量的 $t$ - $\mathrm{BuONa}$ 作碱时, 获得了目标物 2-(2-氧代-2-苯基乙 基)-4H-苯并噻喃-4-酮(3a), 产率 63\% (Entry 4). 接着, 2 倍量的配体邻菲罗啉的加入显著提高了反应产率，达到 $82 \%$ (Entry 5). 于是，另外 2 种常用铜配体 $N, N$-四甲基 乙二胺 $(\mathbf{L} 2)$ 和 $L$-脯氨酸 $(\mathbf{L} 3)$ 也被考察, 但结果都不如邻 菲罗啉(L1) (Entries 6, 7). 随后对碱进行了笁选, 结果 显示: 碱性稍弱的 $t$-BuOK、NaOEt、 $\mathrm{Cs}_{2} \mathrm{CO}_{3}$ 和碱性更强 
表 2 铜催化合成化合物 $\mathbf{3 a}$ 的反应条件优化 ${ }^{a}$

Table 2 Cu-catalyzed reaction condition optimization for $\mathbf{3 a}$

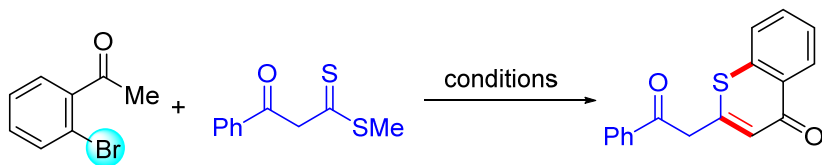

$1 \mathrm{a}$

$2 a$

3a

\begin{tabular}{|c|c|c|c|c|c|c|}
\hline Entry & $\mathrm{Cu}$ source & $\mathbf{L}$ (equiv.) & Base (equiv.) & Solvent & $T /{ }^{\circ} \mathrm{C}$ & Isolated yield $/ \%$ \\
\hline 1 & - & - & - & Toluene & 80 & NP \\
\hline 2 & $\mathrm{CuI}$ & - & - & Toluene & 80 & NP \\
\hline 3 & - & - & $t$-BuONa & Toluene & 80 & NP \\
\hline 4 & $\mathrm{CuI}$ & - & $t-\mathrm{BuONa}$ & Toluene & 80 & 63 \\
\hline 5 & $\mathrm{CuI}$ & L1 (2) & $t$-BuONa & Toluene & 80 & 82 \\
\hline 6 & $\mathrm{CuI}$ & L2 (2) & $t-\mathrm{BuONa}$ & Toluene & 80 & 72 \\
\hline 7 & $\mathrm{CuI}$ & L3 (2) & $t$-BuONa & Toluene & 80 & 76 \\
\hline 8 & $\mathrm{CuI}$ & L1 (2) & $t$-BuOK & Toluene & 80 & 70 \\
\hline 9 & $\mathrm{CuI}$ & L1 (2) & $\mathrm{NaOEt}$ & Toluene & 80 & 14 \\
\hline 10 & $\mathrm{CuI}$ & L1 (2) & $\mathrm{Cs}_{2} \mathrm{CO}_{3}$ & Toluene & 80 & $\mathrm{NP}$ \\
\hline 11 & $\mathrm{CuI}$ & L1 (2) & $\mathrm{NaH}$ & Toluene & 80 & 65 \\
\hline 12 & $\mathrm{CuI}$ & L1 (2) & $t$-BuONa (3) & Toluene & 80 & 44 \\
\hline 13 & $\mathrm{CuI}$ & L1 (2) & $t$-BuONa (5) & Toluene & 80 & 78 \\
\hline 14 & $\mathrm{CuBr}$ & L1 (2) & $t$-BuONa & Toluene & 80 & 57 \\
\hline 15 & $\mathrm{CuCl}$ & L1 (2) & $t-\mathrm{BuONa}$ & Toluene & 80 & Trace \\
\hline 16 & $\mathrm{Cu}_{2} \mathrm{O}$ & L1 (2) & $t$-BuONa & Toluene & 80 & 56 \\
\hline 17 & CuI & L1 (1) & $t$-BuONa & Toluene & 80 & $82(91)^{b}$ \\
\hline 18 & $\mathrm{CuI}$ & L1 (1) & $t$-BuONa & $\mathrm{DMF}$ & 80 & 25 \\
\hline 19 & $\mathrm{CuI}$ & L1 (1) & $t-\mathrm{BuONa}$ & $\mathrm{MeCN}$ & 80 & 20 \\
\hline 20 & $\mathrm{CuI}$ & L1 (1) & $t-\mathrm{BuONa}$ & DCE & 80 & 10 \\
\hline 21 & $\mathrm{CuI}$ & L1 (1) & $t-\mathrm{BuONa}$ & $\mathrm{EtOH}$ & 80 & 18 \\
\hline 22 & $\mathrm{CuI}$ & L1 (1) & $t$-BuONa & Toluene & 70 & 66 \\
\hline 23 & $\mathrm{CuI}$ & L1 (1) & $t$-BuONa & Toluene & 90 & 29 \\
\hline 24 & $\mathrm{CuI}$ & L1 (1) & $t-\mathrm{BuONa}$ & Toluene & 80 & $34^{c}$ \\
\hline 25 & $\mathrm{CuI}$ & L1 (1) & $t$-BuONa & Toluene & 80 & $61^{d}$ \\
\hline
\end{tabular}

${ }^{a}$ 除特别说明外, 反应条件为: 1a (2 equiv.), $2 \mathrm{a}(0.5 \mathrm{mmol}), \mathrm{Cu}$ salt (1 equiv.), 碱(4 equiv.), 溶剂 $(1.5 \mathrm{~mL})$, 氮气下反应 $4 \mathrm{~h} ;{ }^{b}$ 使用邻碘苯乙酮, 反应时间 $1.5 \mathrm{~h}$; ${ }^{c} \mathrm{CuI}(0.5$ equiv. $){ }^{d}$ 在空气中反应.

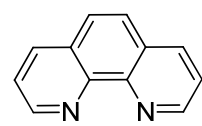

L1<smiles>CN(C)CCN(C)C</smiles>

L2<smiles>O=C(O)C1CCCN1</smiles>

L3
的 $\mathrm{NaH}$, 均给出更低的产率, 甚至无产物生成(Entries $8 \sim 11)$. 确定了 $t$-BuONa 为最佳碱后, 碱量被研究, 实 验结果表明更少的碱对该反应不利(Entry 12); 而更多 的碱也没能改善反应的产率(Entry 13). 随后, 考察了其 它常用的一价铜盐如 $\mathrm{CuBr} 、 \mathrm{CuCl} 、 \mathrm{Cu}_{2} \mathrm{O}$, 但它们给出 的产率都低于 $\mathrm{CuI}$ (Entries 14 16). 有意思的是, 当配 体 L1 的量降低为 1 倍时, 仍然获得了很好的结果(Entry 17). 其它溶剂如 $N, N$-二甲基甲酰胺(DMF)、乙腈、二氯 乙烷和乙醇均使产率降低(Entries 18～21). 降低反应温 度, 产率略微降低, 升高反应温度目标物产率降低至 $29 \%$ ，原料邻溴苯乙酮大量生成自身缩合产物(Entries $22,23)$. 最后, 尝试降低催化剂碘化亚铜的用量为 0.5
倍时, 产率降低为 34\% (Entry 24). 在空气中的对照实 验表明, 该反应需要 $\mathrm{N}_{2}$ 保护. 最终, 确定该反应的最佳 条件是: $\mathrm{CuI} /$ 邻菲罗啉(1 equiv.)作促进剂, $t$ - $\mathrm{BuONa}$ (4 equiv.)作碱, 甲苯作溶剂, $80{ }^{\circ} \mathrm{C}, \mathrm{N}_{2}$ 保护下反应 $4 \mathrm{~h}$ (Entry 17).

值得一提的是，使用邻碘苯乙酮替代邻溴苯乙酮进 行该反应时, 3a 的产率达到 91\%, 同时反应时间也缩短 至 $1.5 \mathrm{~h}$ (Entry 17); 相反, 使用邻氯苯乙酮时, 反应变得 复杂, 只观察到少量的 3a. 考虑到碘代物价格较溴代物 高, 本文均采用取代邻溴苯乙酮进行研究.

\section{2 底物拓展}

为了考察该合成方法的普遍适用性, 我们在上述最 佳反应条件下进行了底物拓展研究(表 3 ). 从表 3 可以看 出, 对于底物 $\beta$-羰基二硫代羧酸酯 $(2), \mathrm{R}^{2}$ 为脂肪族取代 基(例如 $3 n$ 和 $3 u$ )或杂环取代基如噻吩环和呋喃环(例如 31 和 $3 \mathrm{~m}$ ) 时, 产物 3 的产率明显低于芳香族取代基. 而 
表 3 化合物 $\mathbf{3}$ 的合成 ${ }^{a, b}$

Table 3 Synthesis of compounds 3

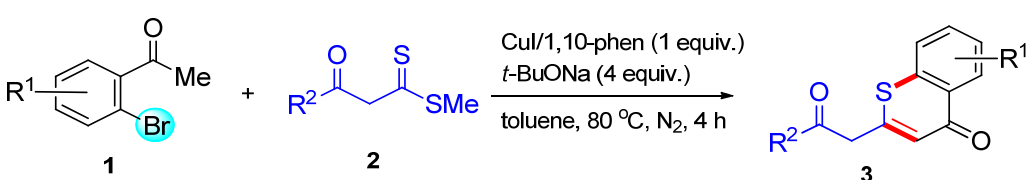<smiles>O=C(Cc1cc(=O)c2ccccc2s1)c1ccccc1</smiles>

$3 a, 82 \%$<smiles>O=C(Cc1cc(=O)c2ccccc2s1)c1ccccc1Cl</smiles>

3b, $61 \%$<smiles>O=C(Cc1cc(=O)c2ccccc2s1)c1cccc(Cl)c1</smiles>

$3 c, 84 \%$<smiles>O=C(Cc1cc(=O)c2ccccc2s1)c1ccc(Cl)cc1</smiles>

$3 d, 81 \%$<smiles>O=C(Cc1cc(=O)c2ccccc2s1)c1ccc(Br)cc1</smiles>

$3 e, 77 \%$<smiles>COc1ccc(C(=O)Cc2cc(=O)c3ccccc3s2)cc1</smiles>

3f, $79 \%$

3g, $36 \%$

3h, $86 \%$

$3 \mathbf{i}, 88 \%$

3j, $87 \%$<smiles>O=C(Cc1cc(=O)c2ccccc2s1)c1ccc(Cl)cc1Cl</smiles>

3k, $72 \%$<smiles>O=C(O)c1ccccc1C(=O)Cc1cccs1</smiles>

$3 \mathrm{I}, 68 \%$<smiles>O=C(CC1C=CC(=O)c2ccccc2S1)c1ccco1</smiles>

$3 \mathrm{~m}, 61 \%$<smiles>CC(=O)Cc1cc(=O)c2ccccc2s1</smiles>

3n, $53 \%$<smiles>O=C(Cc1cc(=O)c2cc(F)ccc2s1)c1ccccc1</smiles>

3o, $81 \%$<smiles>O=C(Cc1cc(=O)c2cc(F)ccc2s1)c1ccc(Br)cc1</smiles><smiles>O=C(Cc1cc(=O)c2cc(F)ccc2s1)c1ccc([N+](=O)[O-])cc1</smiles>

$3 q, 83 \%$<smiles>O=C1CCCC1c1cc(=O)c2ccccc2s1</smiles>

$3 u$, trace

$\overline{{ }^{a}}$ 反应条件: $1(1.0 \mathrm{mmol}), 2(0.5 \mathrm{mmol}), \mathrm{CuI}(0.5 \mathrm{mmol})$, 邻菲罗啉 $(0.5 \mathrm{mmol}), t-\mathrm{BuONa}(2 \mathrm{mmol})$, 甲苯 $(1.5 \mathrm{~mL}), 80{ }^{\circ} \mathrm{C}$, 氮气下反应 $4 \mathrm{~h} ;{ }^{b}$ 分离后的产率.

且, 苯环上在对位连有供电子基时, 其活性要远远大于 带有吸电子基的活性, 其中苯环连有强吸电子的三氟甲 基时产率低至 36\% (3g). 而且, 2,4-二取代 $\beta$-羰基二硫代 羧酸酯的反应也能顺利进行, 并给出较好的产率 $(3 \mathbf{j}$ 和 $3 \mathbf{k})$. 然而, 邻氯取代的 $\beta$-羰基二硫代羧酸酯的反应产 率较低(3b). 对于底物取代邻溴苯乙酮 1, 当苯环上带 有吸电子基如氟原子时，产率略有降低(3o).

所有产物结构均经 IR、 ${ }^{1} \mathrm{H}$ NMR、 ${ }^{13} \mathrm{C}$ NMR、HRMS 方法表征.

\section{3 可能的反应机理}

基于以上的实验结果, 我们推测了该反应可能的机 理, 如 Scheme 3 所示.

$\beta$-羰基二硫代羧酸酯 (2) 的活泼亚甲基在强碱 $t$-BuONa 作用下被夺氢, 生成稳定的负离子中间体 $\mathbf{A}$, 负电荷由碳原子转移到硫原子上, 与碘化亚铜和配体邻 菲罗啉形成的配合物 $\mathbf{L}-\mathrm{Cu}-\mathrm{I}$ 作用, 脱去碘负离子, 形成底物 2 参与的配合物中间体 $\mathbf{B}^{[10 \mathrm{a}]}, \mathbf{B}$ 随后与底物 $\mathbf{1}$
发生氧化加成，生成中间体 $\mathbf{C}$, 随后 $\mathbf{C}$ 发生还原反应, 同时消除一价铜的溴配合物 $\mathbf{L}-\mathrm{Cu}-\mathrm{Br}$, 得到中间体 $\mathbf{D}$. 在强碱作用下，该中间体 $\mathbf{D}$ 的邻溴苯乙酮片段中的甲基 失去质子得到碳负阴离子 $\mathbf{E}$ ，它接着发生分子内的亲核 取代生成中间体 4 , 同时失去一分子 $\mathrm{CH}_{3} \mathrm{SH}$. 最后经 1,3-H 迁移得到目标物 3 .

\section{2 结论}

我们发展了一种铜催化 $\beta$-羰基二硫代羒酸酯和邻 溴苯乙酮经 Ullmann 偶联反应，高效合成硫代色酮类衍 生物的合成方法. 该方法一步构建了一个 $\mathrm{C}-\mathrm{S}$ 键和一 个 $\mathrm{C}=\mathrm{C}$ 键, 相信该合成方法将在有机化学和药物化学 方面得到很好的应用.

\section{3 实验部分}

\section{1 仪器与试剂}

Nicolet iS10 傅立叶变换红外光谱仪, $\mathrm{KBr}$ 压片; 


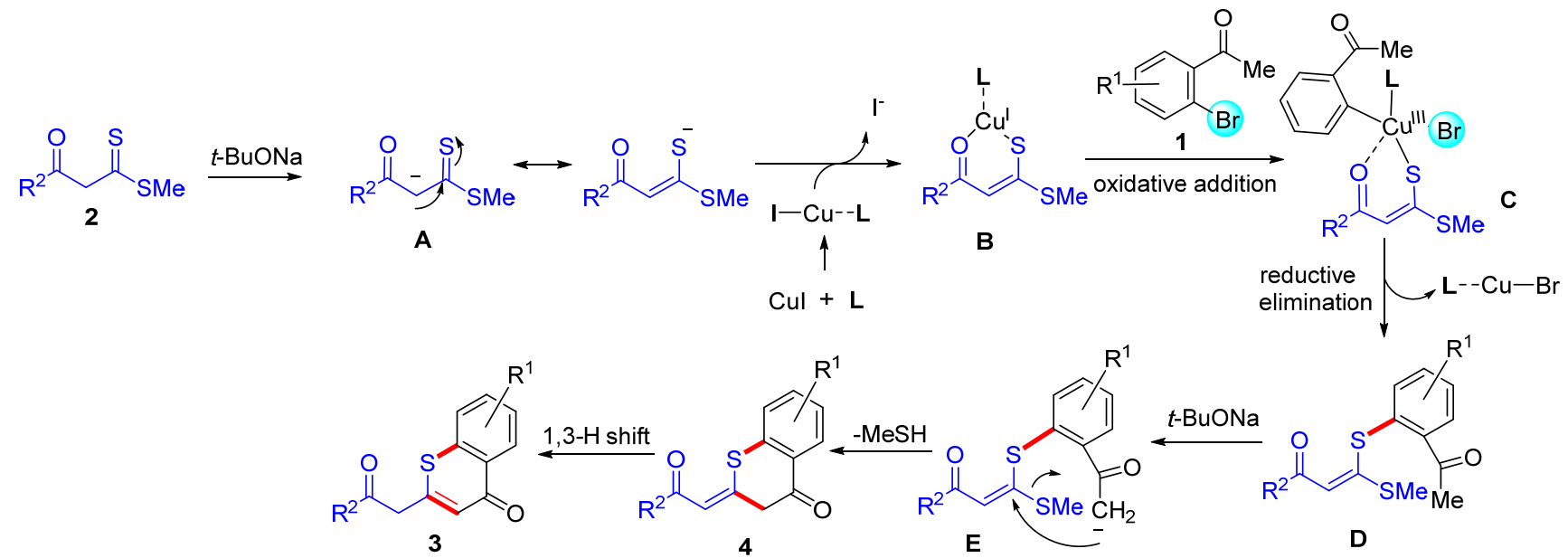

图式 3 可能的铜催化反应机理

Scheme 3 Possible copper-catalyzed reaction mechanism

Bruker AC-500 型核磁共振仪, TMS 为内标, $\mathrm{CDCl}_{3}$ 为溶 剂; Bruker Esquire Hct 质谱仪, ESI 源(电喷雾离子源); EYELA, N-1100 型旋转蒸发器(上海爱朗仪器有限公 司); DLSB-10/40 型低温冷却液循环原(巩义市予华仪器 有限责任公司); RY-1 型毛细管显微熔点仪, 天津分析仪 器厂, 温度计未校正; 薄层色谱(TLC, Thin layer chromatography)所用硅胶为 GF254, 青岛海洋化工厂产品, 紫外 $254 \mathrm{~nm}$ 或 $365 \mathrm{~nm}$ 观察. 所有溶剂和试剂均为分析 纯.

\section{2 实验方法}

\subsection{1 $\beta$-羰基二硫代羧酸酯(2)的合成}

参考文献[12]合成, 以无取代 $\beta$-羰基二硫代羧酸酯 (2a)为例. 向 $125 \mathrm{~mL}$ 三口瓶中加入无取代苯乙酮 $1.20 \mathrm{~g}$ (10 mmol), 及经无水处理的 $N, N$-二甲基甲酰胺(DMF) 6 $\mathrm{mL}$ 和正已烷 $24 \mathrm{~mL}$, 然后加入氢化钠 $0.8 \mathrm{~g}(20 \mathrm{mmol}$, $60 \%$ ), 常温搅拌 $5 \mathrm{~min}$ 之后, 缓慢滴加三硫代碳酸二甲 酯 $1.38 \mathrm{~g}(10 \mathrm{mmol})$, 在 $5 \mathrm{~min}$ 内滴完, 继续常温摚拌 $4 \mathrm{~h}$ 至反应完成, 将上层正已烷尽量倒出, 剩余物倒入冰水 中 $(20 \mathrm{~mL})$, 用稀盐酸 $(2 \mathrm{~mol} / \mathrm{L})$ 调 $\mathrm{pH}=2 \sim 3$, 用二氯甲 烷 $(20 \mathrm{~mL} \times 3)$ 萃取, 合并有机相, 有机相先用饱和食盐 水洗后再用无水硫酸镁干燥, 抽滤, 将滤液旋蒸, 柱层 析(石油醚作展开剂)分离得黄色固体 $\mathbf{2 a}^{[12 \mathrm{a}]}$, 产率 $80 \%$.

\subsection{2 硫代色酮类衍生物 $\mathbf{3}$ 的合成}

以 2-(2-氧代-2-苯基乙基)- $4 H$-苯并噻喃-4-酮(3a)为 例. 称取苯甲酰基二硫代乙酸甲酯(2a) $(0.5 \mathrm{mmol}, 0.105$ g), CuI $(0.5 \mathrm{mmol}, 0.095 \mathrm{~g})$, 邻菲罗啉(L1) $(0.5 \mathrm{mmol}$, $0.0901 \mathrm{~g}), t$-BuONa $(2.0 \mathrm{mmol}, 0.192 \mathrm{~g})$ 于史莱克管中, 充换氮气三次, 用注射器加入溶剂甲苯 $(1.5 \mathrm{~mL}), 80{ }^{\circ} \mathrm{C}$ 搅拌, 称取邻溴苯乙酮(1a) $(1.0 \mathrm{mmol}, 0.190 \mathrm{~g})$ 缓慢滴入 体系, $30 \mathrm{~min}$ 滴完, 继续反应, 至 TLC 检测 $\mathbf{2 a}$ 反应完全
后终止反应, 加水 $(10 \mathrm{~mL})$, 用稀盐酸 $(1 \mathrm{~mol} / \mathrm{L})$ 调至弱酸 性, 用乙酸乙酯 $(10 \mathrm{~mL} \times 3)$ 萃取, 合并有机相, 有机相 盐洗后用无水硫酸镁干燥, 抽滤, 将滤液旋蒸, 柱层析 $\left(V_{\text {石油敷 }}: V_{\text {乙酸乙䤃 }}=6: 1 \sim 1: 1\right.$ 作展开剂梯度洗脱 $)$ 分离得 黄色固体 3a.

\subsection{3 化合物 $\mathbf{3 a} \sim \mathbf{3 q}$ 的物理性质和光谱数据如下:}

2-(2-氧代-2-苯乙基)- $4 H$-苯并噻喃-4-酮(3a): $115 \mathrm{mg}$ 黄色固体, 产率 $82 \%$. m.p. $122 \sim 123{ }^{\circ} \mathrm{C} ;{ }^{1} \mathrm{H}$ NMR $\left(\mathrm{CDCl}_{3}, 500 \mathrm{MHz}\right) \delta: 4.31(\mathrm{~s}, 2 \mathrm{H}), 6.94(\mathrm{~s}, 1 \mathrm{H}), 7.49 \sim$ $7.52(\mathrm{~m}, 3 \mathrm{H}), 7.56 \sim 7.59(\mathrm{~m}, 2 \mathrm{H}), 7.62(\mathrm{t}, J=7 \mathrm{~Hz}, 1 \mathrm{H})$, $8.01(\mathrm{~d}, J=8 \mathrm{~Hz}, 2 \mathrm{H}), 8.49(\mathrm{~d}, J=8 \mathrm{~Hz}, 1 \mathrm{H}) ;{ }^{13} \mathrm{C}$ NMR $\left(\mathrm{CDCl}_{3}, 125 \mathrm{MHz}\right) \delta: 46.3,126.2,126.9,127.7,128.5$, $128.9,130.0,130.7,131.5,134.0,135.7,137.6,147.6$, 180.3, 193.6; IR (KBr) v: 3061, 2916, 1686, 1625, 1594, 1439, 1332, 1215, 778, 750, 684, $638 \mathrm{~cm}^{-1}$; HRMS (ESI-TOF) calcd for $\mathrm{C}_{17} \mathrm{H}_{13} \mathrm{O}_{2} \mathrm{~S}[\mathrm{M}+\mathrm{H}]^{+}$281.0636, found 281.0641 .

2-[2-(2-氯苯基)-2-氧代乙基]- $4 H$-苯并噻喃-4-酮 (3b): $96 \mathrm{mg}$ 黄色固体, 产率 61\%. m.p. $97 \sim 98{ }^{\circ} \mathrm{C} ;{ }^{1} \mathrm{H}$ NMR $\left(\mathrm{CDCl}_{3}, 500 \mathrm{MHz}\right) \delta: 4.32(\mathrm{~s}, 2 \mathrm{H}), 6.92(\mathrm{~s}, 1 \mathrm{H})$, $7.34 \sim 7.37(\mathrm{~m}, 1 \mathrm{H}), 7.44 \sim 7.46(\mathrm{~m}, 2 \mathrm{H}), 7.52 \sim 7.53(\mathrm{~m}$, $2 \mathrm{H}), 7.57 \sim 7.60(\mathrm{~m}, 2 \mathrm{H}), 8.50(\mathrm{~d}, J=8 \mathrm{~Hz}, 1 \mathrm{H}) ;{ }^{13} \mathrm{C} \mathrm{NMR}$ $\left(\mathrm{CDCl}_{3}, 125 \mathrm{MHz}\right) \delta: 50.1,126.2,127.1,127.2,127.8$, $128.6,129.6,130.7,131.6,132.7,137.6,137.8,146.8$, 180.4, 196.6; IR (KBr) v: 3141, 1692, 1623, 1591, 1439, 1349, 1204, 1102, 976, 784, $746 \mathrm{~cm}^{-1}$; HRMS (ESI-TOF) calcd for $\mathrm{C}_{17} \mathrm{H}_{12} \mathrm{O}_{2} \mathrm{SCl}[\mathrm{M}+\mathrm{H}]^{+}$315.0247, found 315.0250 .

2-[2-(3-氯苯基)-2-氧代乙基]- $4 \mathrm{H}$-苯并噻喃-4-酮 (3c): $133 \mathrm{mg}$ 黄色固体, 产率 $84 \%$. m.p. $117 \sim 118{ }^{\circ} \mathrm{C} ;{ }^{1} \mathrm{H}$ 
NMR $\left(\mathrm{CDCl}_{3}, 500 \mathrm{MHz}\right) \delta: 4.29(\mathrm{~s}, 2 \mathrm{H}), 6.93(\mathrm{~s}, 1 \mathrm{H}), 7.46$ (t, $J=8 \mathrm{~Hz}, 1 \mathrm{H}), 7.52 \sim 7.55(\mathrm{~m}, 1 \mathrm{H}), 7.57 \sim 7.62(\mathrm{~m}, 3 \mathrm{H})$, 7.89 (d, $J=8 \mathrm{~Hz}, 1 \mathrm{H}), 7.98$ (t, $J=2 \mathrm{~Hz}, 1 \mathrm{H}), 8.50$ (d, $J=8$ $\mathrm{Hz}, 1 \mathrm{H}) ;{ }^{13} \mathrm{C} \mathrm{NMR}\left(\mathrm{CDCl}_{3}, 125 \mathrm{MHz}\right) \delta: 46.3,126.2$, 126.6, 127.1, 127.8, 128.6, 128.6, 130.3, 130.7, 131.6, 134.0, 135.4, 137.2,137.4,146.9,180.3, 192.4; IR (KBr) $v$ : 3078, 2909, 1684, 1607, 1591, 1345, 1211, 778, $732 \mathrm{~cm}^{-1}$; HRMS (ESI-TOF) calcd for $\mathrm{C}_{17} \mathrm{H}_{12} \mathrm{O}_{2} \mathrm{SCl}[\mathrm{M}+\mathrm{H}]^{+}$ 315.0247, found 315.0253 .

2-[2-(4-氯苯基)-2-氧代乙基]- $4 \mathrm{H}$-苯并噻喃-4-酮 (3d): $128 \mathrm{mg}$ 黄色固体, 产率 $81 \%$. m.p. $166 \sim 167{ }^{\circ} \mathrm{C} ;{ }^{1} \mathrm{H}$ $\mathrm{NMR}\left(\mathrm{CDCl}_{3}, 500 \mathrm{MHz}\right) \delta: 4.28(\mathrm{~s}, 2 \mathrm{H}), 6.92(\mathrm{~s}, 1 \mathrm{H}), 7.48$ (d, $J=9 \mathrm{~Hz}, 2 \mathrm{H}), 7.53$ (t, $J=7 \mathrm{~Hz}, 1 \mathrm{H}), 7.56 \sim 7.61(\mathrm{~m}$, $2 \mathrm{H}), 7.95(\mathrm{~d}, J=9 \mathrm{~Hz}, 2 \mathrm{H}), 8.50(\mathrm{~d}, J=8 \mathrm{~Hz}, 1 \mathrm{H}) ;{ }^{13} \mathrm{C}$ NMR $\left(\mathrm{CDCl}_{3}, 125 \mathrm{MHz}\right) \delta: 46.2,126.2,127.0,127.8$, $128.6,129.3,129.9,130.7,131.6,133.9,137.4,140.7$, 147.2, 180.3, 192.4; IR (KBr) v: 3070, 2919, 1687, 1621, 1592, 1438, 1213, 1087, 986, 832, 776, $736 \mathrm{~cm}^{-1}$; HRMS (ESI-TOF) calcd for $\mathrm{C}_{17} \mathrm{H}_{12} \mathrm{O}_{2} \mathrm{SCl}[\mathrm{M}+\mathrm{H}]^{+}$315.0247, found 315.0242 .

2-[2-(4-溴苯基)-2-氧代乙基]-4H-苯并噻喃-4-酮 (3e): $139 \mathrm{mg}$ 黄色固体, 产率 77\%. m.p. $165 \sim 166{ }^{\circ} \mathrm{C} ;{ }^{1} \mathrm{H}$ $\operatorname{NMR}\left(\mathrm{CDCl}_{3}, 500 \mathrm{MHz}\right) \delta$ : $4.27(\mathrm{~s}, 2 \mathrm{H}), 6.92(\mathrm{~s}, 1 \mathrm{H}), 7.52$ $(\mathrm{d}, J=7 \mathrm{~Hz}, 1 \mathrm{H}), 7.56 \sim 7.60(\mathrm{~m}, 3 \mathrm{H}), 7.65(\mathrm{~d}, J=9 \mathrm{~Hz}$, 2H), $7.87(\mathrm{~d}, J=9 \mathrm{~Hz}, 2 \mathrm{H}), 8.49(\mathrm{~d}, J=8 \mathrm{~Hz}, 1 \mathrm{H}),{ }^{13} \mathrm{C}$ $\mathrm{NMR}\left(\mathrm{CDCl}_{3}, 125 \mathrm{MHz}\right) \delta$ : 45.2, 125.2, 126.0, 126.9, $127.7,128.5,129.0,129.8,130.6,131.3,133.4,136.5$, 146.2, 179.3, 191.6; IR (KBr) v: 3062, 2919, 2852, 1688, 1622, 1587, 1438, 1330, 1211, 1072, 987, 777, 735, 594 $\mathrm{cm}^{-1}$; HRMS (ESI-TOF) calcd for $\mathrm{C}_{17} \mathrm{H}_{12} \mathrm{O}_{2} \mathrm{SBr}[\mathrm{M}+$ $\mathrm{H}]^{+}$358.9741, found 358.9752 .

2-[2-(4-氟苯基)-2-氧代乙基]- $4 \mathrm{H}$-苯并噻喃-4-酮 (3f): $118 \mathrm{mg}$ 黄色固体, 产率 79\%. m.p. $139 \sim 140{ }^{\circ} \mathrm{C} ;{ }^{1} \mathrm{H}$ NMR $\left(\mathrm{CDCl}_{3}, 500 \mathrm{MHz}\right) \delta$ : $4.28(\mathrm{~s}, 2 \mathrm{H}), 6.93(\mathrm{~s}, 1 \mathrm{H}), 7.18$ (t, $J=9 \mathrm{~Hz}, 2 \mathrm{H}), 7.51 \sim 7.54(\mathrm{~m}, 1 \mathrm{H}), 7.57 \sim 7.62(\mathrm{~m}, 2 \mathrm{H})$, $8.05(\mathrm{dd}, J=9,5 \mathrm{~Hz}, 2 \mathrm{H}), 8.50(\mathrm{~d}, J=8 \mathrm{~Hz}, 1 \mathrm{H}) ;{ }^{13} \mathrm{C}$ $\operatorname{NMR}\left(\mathrm{CDCl}_{3}, 125 \mathrm{MHz}\right) \delta: 45.3,115.2\left(\mathrm{~d},{ }^{2} J_{\mathrm{C}-\mathrm{F}}=22 \mathrm{~Hz}\right)$, 125.2, 126.0, 126.8, 127.6, 129.7, 130.3, 130.4, 130.6, 131.1, 136.5, 146.5, $165.3\left(\mathrm{~d},{ }^{1} J_{\mathrm{C}-\mathrm{F}}=257 \mathrm{~Hz}\right), 179.4$, 191.1; IR (KBr) v: 3063, 2949, 2916, 1687, 1625, 1594, 1552, 1509, 1438, 1329, 1241, 1211, 1158, 990, 831, 733 $\mathrm{cm}^{-1}$; HRMS (ESI-TOF) calcd for $\mathrm{C}_{17} \mathrm{H}_{12} \mathrm{O}_{2} \mathrm{SF}[\mathrm{M}+\mathrm{H}]^{+}$ 299.0542, found 299.0553.

2-[2-(4-三氟甲基苯基)-2-氧代乙基]-4H-苯并噻喃4-酮(3g): $63 \mathrm{mg}$ 黄色固体, 产率 36\%. m.p. 187 $189{ }^{\circ} \mathrm{C}$;
${ }^{1} \mathrm{H}$ NMR $\left(\mathrm{CDCl}_{3}, 500 \mathrm{MHz}\right) \delta: 4.34$ (s, 2H), $6.94(\mathrm{~s}, 1 \mathrm{H})$, $7.52 \sim 7.55(\mathrm{~m}, 1 \mathrm{H}), 7.57 \sim 7.62(\mathrm{~m}, 2 \mathrm{H}), 7.78(\mathrm{~d}, J=8 \mathrm{~Hz}$, $2 \mathrm{H}), 8.12(\mathrm{~d}, J=8 \mathrm{~Hz}, 2 \mathrm{H}), 8.50(\mathrm{~d}, J=8 \mathrm{~Hz}, 1 \mathrm{H}) ;{ }^{13} \mathrm{C}$ NMR $\left(\mathrm{CDCl}_{3}, 125 \mathrm{MHz}\right) \delta: 46.5,60.4,126.1\left(\mathrm{~d},{ }^{2} J_{\mathrm{C}-\mathrm{F}}=22\right.$ $\mathrm{Hz})$, 127.1, 127.8, 127.9, 128.6, 128.9, 130.4, 130.65, 131.7, 137.3, 138.2, 146.8, 171.2, 180.3, 192.7; IR (KBr) $v: 2926,1689,1612,1594,1439,1411,1327,1125,1112$, 1067, 990, 840, 781, 770, 736, $600 \mathrm{~cm}^{-1}$; HRMS (ESI-TOF) calcd for $\mathrm{C}_{18} \mathrm{H}_{15} \mathrm{O}_{2} \mathrm{SF}_{3}[\mathrm{M}+\mathrm{H}]^{+}$351.0667, found 351.0672 .

2-(2-氧代-2-对甲苯基)-4H-苯并噻喃-4-酮(3h)：127 $\mathrm{mg}$ 黄色固体, 产率 86\%. m.p.134 135 ${ }^{\circ} \mathrm{C} ;{ }^{1} \mathrm{H}$ NMR $\left(\mathrm{CDCl}_{3}, 500 \mathrm{MHz}\right) \delta: 2.42(\mathrm{~s}, 3 \mathrm{H}), 4.28(\mathrm{~s}, 2 \mathrm{H}), 6.94(\mathrm{~s}$, $1 \mathrm{H}), 7.30(\mathrm{~d}, J=8 \mathrm{~Hz}, 2 \mathrm{H}), 7.49 \sim 7.52(\mathrm{~m}, 1 \mathrm{H}), 7.55 \sim$ $7.58(\mathrm{~m}, 2 \mathrm{H}), 7.91(\mathrm{~d}, J=8 \mathrm{~Hz}, 2 \mathrm{H}), 8.49$ (d, $J=8 \mathrm{~Hz}$, $1 \mathrm{H}) ;{ }^{13} \mathrm{C}$ NMR $\left(\mathrm{CDCl}_{3}, 125 \mathrm{MHz}\right) \delta: 21.6,46.2,126.2$, $126.8,127.6,128.5,128.7,129.6,131.4,133.2,137.6$, 145.0, 147.9, 180.3, 193.2; IR (KBr) v: 3051, 2949, 2856, 1682, 1611, 1595, 1343, 810, $777 \mathrm{~cm}^{-1}$; HRMS (ESI-TOF) calcd for $\mathrm{C}_{18} \mathrm{H}_{15} \mathrm{O}_{2} \mathrm{~S}[\mathrm{M}+\mathrm{H}]^{+}$295.0793, found 295.0786.

2-[2-(4-甲氧苯基)-2-氧代乙基]-4H-苯并噻喃-4-酮 (3i): $137 \mathrm{mg}$ 黄色固体, 产率 88\%. m.p. $125 \sim 126{ }^{\circ} \mathrm{C} ;{ }^{1} \mathrm{H}$ $\mathrm{NMR}\left(\mathrm{CDCl}_{3}, 500 \mathrm{MHz}\right) \delta$ : 3.88 (s, 3H), 4.26 (s, 2H), 6.94 (s, $1 \mathrm{H}), 6.96(\mathrm{~d}, J=9 \mathrm{~Hz}, 2 \mathrm{H}), 7.49 \sim 7.53(\mathrm{~m}, 1 \mathrm{H}), 7.56 \sim$ $7.60(\mathrm{~m}, 2 \mathrm{H}), 7.99(\mathrm{~d}, J=9 \mathrm{~Hz}, 2 \mathrm{H}), 8.49(\mathrm{~d}, J=8 \mathrm{~Hz}$, $1 \mathrm{H}) ;{ }^{13} \mathrm{C} \mathrm{NMR}\left(\mathrm{CDCl}_{3}, 125 \mathrm{MHz}\right) \delta: 45.1,54.6,113.2$, $125.2,125.8,126.7,127.6,130.0,130.5,136.7,147.2$, 163.3, 179.4, 191.1; IR (KBr) v: 3065, 3023, 2919, 2852, 1682, 1614, 1601, 1574, 1309, 1261, 1176, 1029, 976, 831, $775 \mathrm{~cm}^{-1}$; HRMS (ESI-TOF) calcd for $\mathrm{C}_{18} \mathrm{H}_{15} \mathrm{O}_{3} \mathrm{~S}$ [M+ $\mathrm{H}]^{+}$311.0742, found 311.0752 .

2-[2-(2,4-二甲基苯基)-2-氧代乙基]-4H-苯并噻喃-4酮(3j): $134 \mathrm{mg}$ 黄色固体, 产率 87\%. m.p. $130 \sim 131{ }^{\circ} \mathrm{C}$; ${ }^{1} \mathrm{H}$ NMR $\left(\mathrm{CDCl}_{3}, 500 \mathrm{MHz}\right) \delta: 2.36(\mathrm{~s}, 3 \mathrm{H}), 2.51(\mathrm{~s}, 3 \mathrm{H})$, $4.24(\mathrm{~s}, 2 \mathrm{H}), 6.91(\mathrm{~s}, 1 \mathrm{H}), 7.09 \sim 7.11(\mathrm{~m}, 2 \mathrm{H}), 7.49 \sim 7.52$ $(\mathrm{m}, 1 \mathrm{H}), 7.55 \sim 7.59(\mathrm{~m}, 2 \mathrm{H}), 7.69(\mathrm{~d}, J=8 \mathrm{~Hz}, 1 \mathrm{H}), 8.49$ $(\mathrm{d}, J=8 \mathrm{~Hz}, 1 \mathrm{H}) ;{ }^{13} \mathrm{C}$ NMR $\left(\mathrm{CDCl}_{3}, 125 \mathrm{MHz}\right) \delta: 20.4$, 20.8, 47.7, 125.2, 125.6, 125.8, 126.7, 127.6, 128.5, 129.8, $130.5,132.1,132.4,136.7,139.1,142.3,147.2,179.4$, 194.9; IR (KBr) v: 3441, 3057, 2922, 1676, 1626, 1593, 1440, 1347, 1323, 1212, 984, 774, $733 \mathrm{~cm}^{-1}$; HRMS (ESI-TOF) calcd for $\mathrm{C}_{19} \mathrm{H}_{17} \mathrm{O}_{2} \mathrm{~S}[\mathrm{M}+\mathrm{H}]^{+}$309.0952, found 309.0952 .

2-[2-(2,4-二氯苯基)-2-氧代乙基]- $4 H$-苯并噻喃-4-酮 (3k): $126 \mathrm{mg}$ 黄色固体, 产率 72\%. m.p. $173 \sim 174{ }^{\circ} \mathrm{C} ;{ }^{1} \mathrm{H}$ 
NMR $\left(\mathrm{CDCl}_{3}, 500 \mathrm{MHz}\right) \delta: 4.31(\mathrm{~s}, 2 \mathrm{H}), 6.91(\mathrm{~s}, 1 \mathrm{H})$, $7.34 \sim 7.36(\mathrm{~m}, 1 \mathrm{H}), 7.49 \sim 7.55(\mathrm{~m}, 3 \mathrm{H}), 7.57 \sim 7.63(\mathrm{~m}$, $2 \mathrm{H}), 8.50(\mathrm{~d}, J=9 \mathrm{~Hz}, 1 \mathrm{H}) ;{ }^{13} \mathrm{C} \mathrm{NMR}\left(\mathrm{CDCl}_{3}, 125 \mathrm{MHz}\right)$ $\delta: 50.0,126.2,127.1,127.7,127.9,128.6,130.7,130.8$, 131.6, 132.3, 135.9, 137.4, 138.6, 146.5, 180.3, 195.3; IR (KBr) $v: 3093,2920,1694,1620,1594,1581,1440,1197$, 1102, 981, $772 \mathrm{~cm}^{-1}$; HRMS (ESI-TOF) calcd for $\mathrm{C}_{17} \mathrm{H}_{10} \mathrm{Cl}_{2} \mathrm{O}_{2} \mathrm{~S}[\mathrm{M}+\mathrm{H}]^{+}$348.9857, found348.9862.

2-[2-氧代-2-(噻吩-2-基)乙基]-4H-苯并噻喃-4-酮 (31): $98 \mathrm{mg}$ 黄色固体, 产率 68\%. m.p. $111 \sim 112{ }^{\circ} \mathrm{C} ;{ }^{1} \mathrm{H}$ NMR $\left(\mathrm{CDCl}_{3}, 500 \mathrm{MHz}\right) \delta: 4.22(\mathrm{~s}, 2 \mathrm{H}), 6.98(\mathrm{~s}, 1 \mathrm{H}), 7.18$ (t, $J=4 \mathrm{~Hz}, 1 \mathrm{H}), 7.50 \sim 7.53(\mathrm{~m}, 1 \mathrm{H}), 7.55 \sim 7.61(\mathrm{~m}, 2 \mathrm{H})$, $7.73(\mathrm{~d}, J=5 \mathrm{~Hz}, 1 \mathrm{H}), 7.84$ (d, $J=4 \mathrm{~Hz}, 1 \mathrm{H}), 8.49$ (d, $J=8$ $\mathrm{Hz}, 1 \mathrm{H}) ;{ }^{13} \mathrm{C}$ NMR $\left(\mathrm{CDCl}_{3}, 125 \mathrm{MHz}\right) \delta: 47.0,126.2$, $126.9,127.8,128.5,130.7,131.6,133.2,135.4,137.5$, 142.7, 147.2, 180.3, 186.2; IR (KBr) v: 3082, 3017, 2921, 1656, 1603, 1590, 1440, 1413, 1348, 1282, 1102, 776, 738, 601, $537 \mathrm{~cm}^{-1}$; HRMS (ESI-TOF) calcd for $\mathrm{C}_{15} \mathrm{H}_{12} \mathrm{O}_{2} \mathrm{~S}_{2}$ $[\mathrm{M}+\mathrm{H}]^{+}$287.0200, found 287.0205.

2-[2-氧代-2-(呋喃-2-基)乙基]-4H-苯并噻喃-4-酮 (3m): $83 \mathrm{mg}$ 黄色固体, 产率 61\%. m.p. 129.5 130 ${ }^{\circ} \mathrm{C}$; ${ }^{1} \mathrm{H}$ NMR $\left(\mathrm{CDCl}_{3}, 500 \mathrm{MHz}\right) \delta: 4.17$ (s, 2H), 6.61 (d, $J=2$ $\mathrm{Hz}, 1 \mathrm{H}), 6.99(\mathrm{~s}, 1 \mathrm{H}), 7.34(\mathrm{~d}, J=4 \mathrm{~Hz}, 1 \mathrm{H}), 7.51 \sim 7.54$ (m, 1H), $7.57 \sim 7.61(\mathrm{~m}, 2 \mathrm{H}), 7.66(\mathrm{~s}, 1 \mathrm{H}), 8.49(\mathrm{~d}, J=8$ $\mathrm{Hz}, 1 \mathrm{H}) ;{ }^{13} \mathrm{C}$ NMR $\left(\mathrm{CDCl}_{3}, 125 \mathrm{MHz}\right) \delta: 46.1,113.0$, $118.8,126.2,126.9,127.7,128.5,130.7,131.6,137.6$, 147.0, 147.4, 151.6, 180.4, 182.3; IR (KBr) v: 3118, 3023, 1660, 1625, 1592, 1467, 1318, 1007, 779, 737, 596, 530 $\mathrm{cm}^{-1}$; HRMS (ESI-TOF) calcd for $\mathrm{C}_{15} \mathrm{H}_{12} \mathrm{O}_{3} \mathrm{~S}[\mathrm{M}+\mathrm{H}]^{+}$ 271.0429, found 271.0432.

2-(2-氧代丙基)- $4 H$-苯并噻喃-4-酮(3n): $58 \mathrm{mg}$ 黄色 固体, 产率 53\%. m.p. $106 \sim 107{ }^{\circ} \mathrm{C} ;{ }^{1} \mathrm{H}$ NMR $\left(\mathrm{CDCl}_{3}\right.$, $500 \mathrm{MHz}) \delta: 2.30(\mathrm{~s}, 3 \mathrm{H}), 3.74(\mathrm{~s}, 2 \mathrm{H}), 6.87(\mathrm{~s}, 1 \mathrm{H})$, $7.51 \sim 7.54(\mathrm{~m}, 1 \mathrm{H}), 7.56 \sim 7.62(\mathrm{~m}, 2 \mathrm{H}), 8.50(\mathrm{~d}, J=8 \mathrm{~Hz}$, $1 \mathrm{H}) ;{ }^{13} \mathrm{C}$ NMR $\left(\mathrm{CDCl}_{3}, 125 \mathrm{MHz}\right) \delta: 29.6,51.3,126.2$, $126.8,127.8,128.6,130.7,131.6,137.4,146.8,180.3$, 201.6; IR (KBr) v: 3066, 2916, 1714, 1626, 1593, 1439, 1348, 1320, 1166, 1100, 776, 739, $532 \mathrm{~cm}^{-1}$; HRMS (ESI-TOF) calcd for $\mathrm{C}_{12} \mathrm{H}_{11} \mathrm{O}_{2} \mathrm{~S}[\mathrm{M}+\mathrm{H}]^{+} 219.0480$, found 219.0482.

7-氟-2-(2-氧代-2-苯乙基)- $4 H$-苯并噻喃-4-酮 (3o): $121 \mathrm{mg}$ 黄色固体, 产率 81\%. m.p. 143.5 144.5 ${ }^{\circ} \mathrm{C} ;{ }^{1} \mathrm{H}$ NMR $\left(\mathrm{CDCl}_{3}, 500 \mathrm{MHz}\right) \delta: 4.31(\mathrm{~s}, 2 \mathrm{H}), 6.91(\mathrm{~s}, 1 \mathrm{H})$, $7.20 \sim 7.26(\mathrm{~m}, 2 \mathrm{H}), 7.52(\mathrm{t}, J=8 \mathrm{~Hz}, 2 \mathrm{H}), 7.64$ (t, $J=7$ $\mathrm{Hz}, 1 \mathrm{H}), 8.01(\mathrm{~d}, J=8 \mathrm{~Hz}, 2 \mathrm{H}), 8.51(\mathrm{dd}, J=9,6 \mathrm{~Hz}, 1 \mathrm{H})$;
${ }^{13} \mathrm{C} \mathrm{NMR}\left(\mathrm{CDCl}_{3}, 125 \mathrm{MHz}\right) \delta: 46.1,112.0\left(\mathrm{~d},{ }^{2} J_{\mathrm{C}-\mathrm{F}}=25\right.$ $\mathrm{Hz}), 116.3\left(\mathrm{~d},{ }^{2} J_{\mathrm{C}-\mathrm{F}}=22 \mathrm{~Hz}\right), 127.1,127.5,128.5,129.0$, $131.7\left(\mathrm{~d},{ }^{3} J_{\mathrm{C}-\mathrm{F}}=8 \mathrm{~Hz}\right), 134.1,135.6,139.7,139.7,147.2$, $164.0\left(\mathrm{~d},{ }^{1} J_{\mathrm{C}-\mathrm{F}}=256 \mathrm{~Hz}\right), 179.3,193.3 ; \mathrm{IR}(\mathrm{KBr}) v: 3060$, 2924, 1697, 1674, 1630, 1604, 1478, 1247, 1214, 1170, 1092, 972, 866, 677, $612 \mathrm{~cm}^{-1}$; HRMS (ESI-TOF) calcd for $\mathrm{C}_{17} \mathrm{H}_{12} \mathrm{O}_{2} \mathrm{FS}[\mathrm{M}+\mathrm{H}]^{+}$299.0542, found 299.0549.

2-[2-(4-澳苯基)-2-氧代乙基]-7-氟-4H-苯并噻喃-4酮(3p): $145 \mathrm{mg}$ 黄色固体, 产率 77\%. m.p. 196 $197{ }^{\circ} \mathrm{C}$; ${ }^{1} \mathrm{H}$ NMR $\left(\mathrm{CDCl}_{3}, 500 \mathrm{MHz}\right) \delta: 4.27$ (s, 2H), $6.89(\mathrm{~s}, 1 \mathrm{H})$, $7.21 \sim 7.25(\mathrm{~m}, 2 \mathrm{H}), 7.66(\mathrm{~d}, J=8 \mathrm{~Hz}, 2 \mathrm{H}), 7.87$ (d, $J=8$ $\mathrm{Hz}, 2 \mathrm{H}), 8.52(\mathrm{dd}, J=8.85,5.90 \mathrm{~Hz}, 1 \mathrm{H}) ;{ }^{13} \mathrm{C} \mathrm{NMR}$ $\left(\mathrm{CDCl}_{3}, 125 \mathrm{MHz}\right) \delta: 45.6,45.7,112.1\left(\mathrm{~d},{ }^{2} J_{\mathrm{C}-\mathrm{F}}=24 \mathrm{~Hz}\right)$, $116.5\left(\mathrm{~d},{ }^{2} J_{\mathrm{C}-\mathrm{F}}=22 \mathrm{~Hz}\right), 127.1,127.4,129.6,130.0,131.7$ $\left(\mathrm{d},{ }^{3} J_{\mathrm{C}-\mathrm{F}}=9 \mathrm{~Hz}\right), 132.4,134.3,139.5,146.8,164.1(\mathrm{~d}$, $\left.{ }^{1} J_{\mathrm{C}-\mathrm{F}}=256 \mathrm{~Hz}\right), 179.3,192.5$; IR (KBr) $v: 3093,1930$, 1681, 1622, 1600, 1587, 1478, 1397, 1243, 1212, 1071, 983, 854, 818, $565 \mathrm{~cm}^{-1}$; HRMS (ESI-TOF) calcd for $\mathrm{C}_{17} \mathrm{H}_{11} \mathrm{O}_{2} \mathrm{FSBr}[\mathrm{M}+\mathrm{H}]^{+}$376.9647, found 376.9649.

7-氟-2-(2-氧代-2-对甲基苯基)-4H-苯并噻喃-4-酮 (3q): $130 \mathrm{mg}$ 黄色固体, 产率 83\%. m.p. $174 \sim 175{ }^{\circ} \mathrm{C} ;{ }^{1} \mathrm{H}$ NMR ( $\left.\mathrm{CDCl}_{3}, 500 \mathrm{MHz}\right) \delta: 2.43$ (s, 3H), 4.27 (s, 2H), 6.90 (s, 1H), $7.19 \sim 7.27(\mathrm{~m}, 2 \mathrm{H}), 7.30$ (d, $J=8 \mathrm{~Hz}, 2 \mathrm{H}), 7.90$ (d, $J=8 \mathrm{~Hz}, 2 \mathrm{H}), 8.50(\mathrm{dd}, J=9,6 \mathrm{~Hz}, 1 \mathrm{H}) ;{ }^{13} \mathrm{C} \mathrm{NMR}$ $\left(\mathrm{CDCl}_{3}, 125 \mathrm{MHz}\right) \delta: 21.7,46.0,112.0\left(\mathrm{~d},{ }^{2} J_{\mathrm{C}-\mathrm{F}}=24 \mathrm{~Hz}\right)$, $116.3\left(\mathrm{~d},{ }^{2} J_{\mathrm{C}-\mathrm{F}}=22 \mathrm{~Hz}\right), 127.0,127.4,128.7,129.7,131.7$, $133.1,139.8,145.2,147.6,164.0\left(\mathrm{~d},{ }^{1} J_{\mathrm{C}-\mathrm{F}}=256 \mathrm{~Hz}\right)$, 179.4, 193.0; IR (KBr) v: 2960, 2920, 1928, 1677, 1624, 1604, 1477, 1207, 1093, 979, 854, 814, $542 \mathrm{~cm}^{-1}$; HRMS (ESI-TOF) calcd for $\mathrm{C}_{18} \mathrm{H}_{14} \mathrm{O}_{2} \mathrm{FS}[\mathrm{M}+\mathrm{H}]^{+}$313.0699, found 313.0692 .

辅助材料(Supporting Information) 化合物 2a 的 IR 谱 图及合成的所有新化合物的的 ${ }^{1} \mathrm{H} N M R 、{ }^{13} \mathrm{C} N M R$ 谱图. 这些材料可以免费从本刊网站(http://sioc-journal.cn/)上 下载.

\section{References}

[1] Nakazumi, H.; Ueyama, T.; Kitao, T. J. Heterocycl. Chem. 1985, 22, 1593.

[2] Hoettecke, N.; Rotzoll, S.; Albrecht, U.; Lalk, M.; Fischer, C.; Langer, P. Bioorg. Med. Chem. 2008, 16, 10319.

[3] Razdan, R. K.; Bruni, R. J.; Mehta, A. C.; Weinhardt, K. K.; Papanastassiou, Z. B. J. Med. Chem. 1978, 21, 643.

[4] Dhanak, D.; Keenan, R. M.; Burton, G.; Kaura, A.; Darcy, M. G.; Shah, D. H.; Ridgers, L. H.; Breen, A.; Lavery, P.; Tew, D. G.; West, A. Bioorg. Med.Chem. Lett. 1998, 8, 3677.

[5] (a) Nakib, T. A.; Bezjak, V.; Meegam, M. J.; Chandy, R. Eur. J. 
Med. Chem. 1990, 25, 455.

(b) Gotoda, S.; Takahashi, N.; Nakagawa, H.; Murakami, M.; Takechi, T.; Komura, T.; Uchida, T.; Takagi, Y. Pestic. Sci. 1998, 52, 309 .

(c) Dhanak, D.; Keenen, R. M.; Burton, G.; Kaura, A.; Darcy, M. G.; Shah, D. H.; Ridgers, L. H.; Breen, A.; Lavery, P.; Tew, D. G.; West, A. Bioorg. Med. Chem. Lett. 1998, 8, 3677.

(d) Wang, H.-K.; Bastow, K. F.; Cosentino, L. M.; Lee, K. H. J. Med. Chem. 1996, 39, 1975.

(e) Charris, J.; Dominguez, J.; Labo, G.; Riggione, F. Pharm. Pharmacol. Commun. 1999, 5, 107.

[6] (a) Kitani, S.; Sugawara, K.; Tsutsumi, K.; Morimoto, T.; Kakiuchi, K. Chem. Commun. 2008, 18, 2103.

(b) Zhang, Y.-L.; Tanimoto, H.; Nishiyama, Y.; Morimoto, T.; Kakiuchi, K. Synlett 2012, 23, 367.

(c) Sugiura, R.; Kozaki, R.; Kitani, S.; Gosho, Y.; Tanimoto, H.; Nishiyama, Y.; Morimoto, T.; Kakiuchi, K. Tetrahedron 2013, 69 , 3984 .

(d) Tasuku, I.; Takuya, K.; Seijiro, M. Org. Lett. 2014, 16, 5660.

[7] (a) Inami, T.; Kurahashi, T.; Matsubara, S. Org. Lett. 2014, 16, 5660 .

(b) Sangeetha, S.; Muthupandi, P.; Sekar, G. Org. Lett. 2015, 17, 6006.

(c) Mal, K.; Kaur, A.; Haque, F.; Das, I. J. Org. Chem. 2015, 80, 6400 .

(d) Jenifer Vijay, T. A.; Nandeesh, K. N.; Raghavendra, G. M.; Rangappa, K. S.; Mantelingu, K. Tetrahedron Lett. 2013, 54, 6533. (e) Li, N.-G.; Shi, Z.-H.; Tang, Y.-P.; Ma, H.-Y.; Yang, J.-P.; Li, B.-Q.; Wang, Z.-J.; Song, S.-L.; Duan, J.-A. J. Heterocycl. Chem. 2010, 47, 785 .

(f) Bondock, S.; Metwally, M. A. J. Sulfur Chem. 2008, 29, 623.

(g) Kumar, P.; Rao, A. T.; Pandey, B. J. Chem. Soc., Chem. Commun. 1992, 21, 1580.

(h) Wang, H.-K.; Bastow, K. F.; Cosentino, L. M.; Lee, K.-H. J. Med. Chem. 1996, 39, 1975.

(i) Kataoka, T.; Watanabe, S.; Mori, E.; Kadomoto, R.; Tanimura, S.; Kohno, M. Bioorg. Med. Chem. 2004, 12, 2397.

(g) Zhou, C.-X.; Dubrovsky, A. V.; Larock, R. C. J. Org. Chem.
2006, 71, 1626.

[8] (a) Evano, G.; Blanchard, N.; Toumi, M. Chem. Rev. 2008, 108, 3054 .

(b) Bariwal, J.; Eycken, E. V. Chem. Soc. Rev. 2013, 42, 9283.

(c) Sun, Y.-Y.; Yi, J.; Lu, X.; Zhang, Z.-Q.; Xiao, B.; Fu, Y. Chem. Commun. 2014, 50, 11060.

(d) Lou, Z.-B.; Zhang, S.; Chen, C.; Pang, X.-L.; Li, M.; Wen, L.-R. Adv. Synth. Catal. 2014, 356, 153.

(e) Yan, X.-Y.; Yang, X.-H.; Xi, C.-J. Catal. Sci. Technol. 2014, 4, 4169.

(f) Wen, L.-R.; Jin, X.-J.; Niu, X.-D.; Li, M. J. Org. Chem. 2015, 80,90 .

(g) Liao, Q.; Yang, X.-H.; Xi, C.-J. J. Org. Chem. 2014, 79, 8507.

(i) Liao, S.; Xi, C.-J. Chin. J. Org. Chem. 2012, 32, 986 (in Chinese).

(廖骞, 席婵娟, 有机化学, 2012, 32, 986.)

(g) Liu, W.; Bi, Y.-L. Chin. J. Org. Chem. 2012, 32, 1041 (in Chinese).

(刘伟，毕艳兰，有机化学, 2012, 32, 1041.)

[9] (a) Singh, M. S.; Nandib, G. C.; Chandaa, T. RSC Adv. 2013, 3, 14183.

(b) Ramulu, B. J.; Nagaraju, A.; Chowdhury, S.; Koley, S.; Singh, M. S. Adv. Synth. Catal. 2015, 357, 530.

(c) Nagaraju, A.; Ramulu, B. J.; Shukla, G.; Srivastava, A.; Verma, G. K.; Raghuvanshi, K.; Singh, M. S. Green Chem. 2015, 17, 950.

(d) Ramulu, B. J.; Koley, S. Org. Biomol. Chem. 2016, 14, 434.

(e) Koley, S.; Chowdhury, S.; Chanda, T.; Ramulu, B. J.; Anand, N.; Singh, M. S. Eur. J. Org. Chem. 2015, 409.

[10] (a) Wen, L.-R.; Yuan, W.-K.; Li, M. J. Org. Chem. 2015, 80, 4942. (b) Wen, L.-R.; Li, Z.-R.; Li, M.; Cao, H. Green Chem. 2012, 14, 707.

[11] Mabe, P. J. C.; Knick, S. L.; Shuler, W. G.; Carlisle, S. S.; Smith, E. A.; Puciaty, A. J.; McFadden, T. M. C.; Potter, C.; Metz, C. R.; Beam, C. F.; Pennington, W. T.; VanDerveer, D. G.; McMillan, C. D. Ind. Eng. Chem. Res. 2015, 54, 7207.

[12] (a) Singh, G.; Bhatacharjee, S. S.; Ila, H.; Junjappa, H. Synthesis 1982, 693.

(b) Satheesh, K. N.; Asokan, C. V. Synth. Commun. 1999, 29, 791.

(Li, L.; Lu, Z.) 